\title{
Anatomical consideration of deep calf veins: application to catheter-directed thrombolysis
}

\author{
Kyu-Ho $\mathrm{Yi}^{1,2} \cdot$ Jong-Jin Lee ${ }^{2,3} \cdot$ Hye-Won Hur ${ }^{2} \cdot$ Hee-Jin $\mathrm{Kim}^{2,4}(\mathbb{C}$
}

Received: 15 June 2021 / Accepted: 16 August 2021 / Published online: 25 August 2021

(c) The Author(s), under exclusive licence to Springer-Verlag France SAS, part of Springer Nature 2021

\begin{abstract}
Purpose An antegrade approach is frequently used in catheter-directed thrombolysis to remove deep-vein thrombosis. However, the antegrade approach is difficult when accessing veins with small diameters; therefore, understanding the variation of deep calf vein is important.

Methods This study measured the diameters and surface areas of the proximal and distal posterior tibial vein, peroneal vein, and anterior tibial vein to determine which are preferable for venous access. This study dissected 132 legs from Korean and Thai cadavers. The proximal and distal posterior tibial vein, peroneal vein, and anterior tibial vein were scanned and measured.

Results The mean diameter and surface area were largest for the proximal tibial vein, at $6.34 \mathrm{~mm}$ and $0.312 \mathrm{~cm}^{2}$, respectively, followed by the anterior tibial vein $\left(5.22 \mathrm{~mm}\right.$ and $\left.0.213 \mathrm{~cm}^{2}\right)$, distal posterior tibial vein $\left(3.29 \mathrm{~mm}\right.$ and $\left.0.091 \mathrm{~cm}^{2}\right)$, and peroneal vein $\left(3.43 \mathrm{~mm}\right.$ and $\left.0.081 \mathrm{~cm}^{2}\right)$. The proximal posterior tibial vein and anterior tibial vein have large diameters and surface areas, which make them ideal for applying an antegrade approach in catheter-directed thrombolysis.

Conclusions The distal posterior tibial vein and peroneal vein are not recommended due to their smaller surface areas and also the anatomical variations therein.
\end{abstract}

Keywords Deep calf vein · Diameter · Posterior tibial vein · Anterior tibial vein $\cdot$ Peroneal vein $\cdot$ Surface area $\cdot$ Deep-vein thrombosis $\cdot$ Catheter-directed thrombolysis

\section{Introduction}

Deep-vein thrombosis (DVT) is a frequent vascular disorder that is associated with high morbidity. The prevalence of DVT has increased to $0.1 \%$ in the general population,

Kyu-Ho Yi and Jong-Jin Lee contributed equally.

Hee-Jin Kim

hjk776@yuhs.ac

1 Wonju Public Health Center, COVID-19 Division, Wonju-Si, Korea

2 Division in Anatomy and Developmental Biology, Department of Oral Biology, Human Identification Research Institute, BK21 FOUR Project, Yonsei University College of Dentistry, Room 6 01, 50-1 Yonsei-ro, Seodaemun-gu, Seoul 03722, Korea

3 Uijeongbu Toxnfill Clinic, Seoul, Korea

4 Department of Materials Science and Engineering, College of Engineering, Yonsei University, Seoul 03722, Korea which is largely attributable to advances in diagnostic methods such as ultrasound scanning [9]. DVTs occur most frequently in the lower legs [22], and 20\% of untreated DVTs advance to proximal veins and cause a pulmonary embolism $[3,26]$. Many clinicians suggest practitioners should consider anatomical variations as a risk factor for DVT. Thereby, anatomical variation of the venous system has been studied and classified from decades ago [1,2]. Recently, a study identified and classified the anatomical variation in the deep calf veins (Fig. 1) [29].

Administering a systemic thrombolytic agent is the conventional therapy for reducing the risk of pulmonary embolism and DVT reoccurrence. However, applying only a systemic thrombolytic agent is associated with post-thrombotic syndrome (PTS) in $20-50 \%$ of treated patients [14]. PTS should be avoided since it is a chronic problem of DVT that includes ulceration, swelling, and dermatosclerosis [10].

Systemic thrombolytic agents should also not be applied alone to patients with high blood pressure, stroke, and arterial aneurysms [14]. In the last few decades, 
Fig. 1 The schematic image of the left side posterior tibial veins and its classification. Type Ia had one proximal posterior tibial vein and two distal posterior tibial veins. Type Ib had one proximal posterior tibial vein and three distal posterior tibial veins. Type IIa had two proximal posterior tibial veins and two distal posterior tibial veins. Type IIb had two proximal posterior tibial veins and three distal posterior tibial veins. PPTV proximal posterior tibial vein, DPTV deep posterior tibial vein)

complementary and combination therapies for catheterdirected thrombolysis (CDT) have been suggested that involve the selective release of thrombolytic agents using intervention catheters. Previous studies have demonstrated that CDT is an effective and safe treatment option for DVT $[12,27]$. The combination therapy of CDT and systemic thrombolytic agents for thrombus elimination is recommended in patients with acute DVT in order to relieve acute symptoms and prevent PTS [18]. The American Heart Association recommended CDT as the primary treatment for patients with acute DVT and for preventing symptom progression and the rapid extension of a thrombus, followed by anticoagulation therapy [13].

CDT has the benefits of the rapid relief of painful symptoms with a smaller amount of thrombolytic agent $[8,25]$. The catheter is inserted proximal or distal to the affected sites in the retrograde or antegrade venous approach, respectively [20]. The venous entrance points of CDT are the posterior tibial vein, anterior tibial vein, popliteal vein, and femoral vein. The venous entrance point is selected by considering anatomical variations in and the diameter of the accessing vein. It is necessary to carefully select the venous entrance point by taking into consideration all of the anatomical pros and cons.

A retrograde venous approach via the femoral vein or popliteal vein has the advantages of a short procedural time due to the large diameter of the vein and the smaller number of anatomical variants. The disadvantages of this approach are the possibility of valvular injury due to the access direction being against the direction of blood flow, insufficient removal of the thrombus, and difficulty of positioning the patient during the procedure [5].

An antegrade venous approach via the anterior tibial vein, posterior tibial vein, or peroneal vein has the advantages of sufficient thrombus clearing, easier positioning, and less valvular damage. Additionally, the antegrade approach has no limitation in clearing the proximal extent of the thrombus, including in cases of an inferior popliteal thrombus, which are often not resolved [5]. The disadvantages of the antegrade approach are the long procedural time needed to obtain access and evaluate veins with small diameters and variations [5].

Since CDT is a fairly new treatment, physicians must focus on more-effective and safer access points and approaches. The diameters of these veins and variations


therein should be carefully considered, since CDT is associated with bleeding complications at the insertion point. The venous anatomy of the distal extremities is 
significantly more unpredictable and complex than those of the corresponding arteries and veins in proximal regions [17].

A previous study revealed the diameters of the popliteal veins that are commonly used for the retrograde approach of CDT [15]. However, no previous study has investigated the diameters of deep calf veins.

This study identified the diameters and surface areas of the posterior tibial vein, anterior tibial vein, and peroneal tibial vein. Knowledge of the diameters and surface areas of these veins will help practitioners to select the best vein when adopting an antegrade approach in CDT.

\section{Methods}

This study was conducted in accordance with the principles outlined in the Declaration of Helsinki. Informed consent and approval were obtained from the families of the cadavers before the dissections were performed. All cadavers used in this study were legally donated and approved from ethics committee of the Surgical Anatomy Education Center, Yonsei University College of Medicine (approval code 19-003; approval date: May 15th, 2019).

This study dissected 132 legs from Korean and Thai cadavers, comprising 42 males and 24 females with a mean age of 69.3 years and an age range 43-96 years. The proximal and distal posterior tibial vein, peroneal vein, and anterior tibial vein were sliced at $2 \mathrm{~cm}$ from their diverging or converging points (Fig. 2). The diameter was determined as the mean values of the measured longitudinal and horizontal values. The measurement was conducted in intraluminal diameters.

Proximal and distal posterior tibial veins were defined in accordance with previous research [29]. According to Yi and $\mathrm{Kim}$, the proximal tibial vein begins from the point where the distal posterior tibial vein and peroneal vein merge, and it turns into the popliteal vein at the point where the anterior and proximal tibial veins merge (Fig. 3). The distal tibial vein starts from the point where the medial and lateral plantar veins merge, and it turns into the proximal posterior tibial vein at the point where it merges with the peroneal vein (Fig. 4).

Sliced specimens were mounted and then scanned (CanoScan LiDE 220), and their diameters and surface areas were measured using standard imaging software (Image J, NIH, Bethesda, MD) (Fig. 5).

\section{Statistical analysis}

Comparisons among the cadavers based on sex were performed using independent $t$ tests, with $\mathrm{p}$ values of $<0.05$

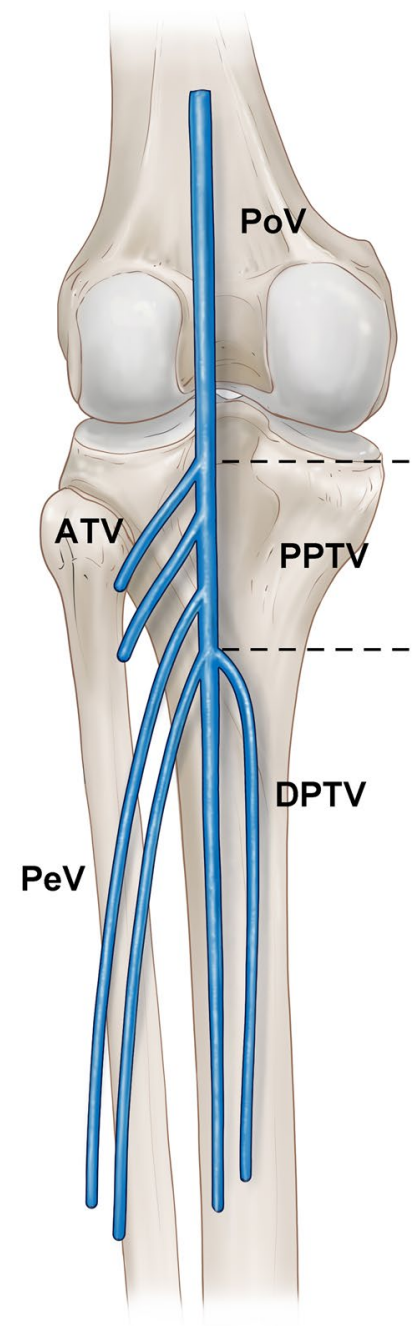

Fig. 2 Schematic of the deep calf veins. Dotted line indicates the proximal posterior tibial vein (PPTV). PoV popliteal vein; $A T V$ anterior tibial vein; $\mathrm{PeV}$ peroneal vein; $D P T V$ distal posterior tibial vein

considered to indicate significant differences. All measured values were analyzed using SPSS software (version 15.0).

\section{Results}

\section{Diameters}

The popliteal, proximal posterior tibial, anterior tibial, peroneal, and distal posterior tibial veins had diameters of $7.42 \pm 1.21 \mathrm{~mm}$ (mean $\pm \mathrm{SD}$, range $5.43-9.58 \mathrm{~mm}$ ), $6.34 \pm 1.51 \mathrm{~mm}$ (range $3.3-8.7 \mathrm{~mm}$ ), $5.22 \pm 1.38 \mathrm{~mm}$ (range $3.40-7.43 \mathrm{~mm}$ ), $3.43 \pm 1.24 \mathrm{~mm}$ (range $1.53-5.64 \mathrm{~mm}$ ), and $3.29 \pm 1.09 \mathrm{~mm}$ (range 1.23-5.37 mm), respectively (Fig. 4). There were no sex-related differences in the diameters. 

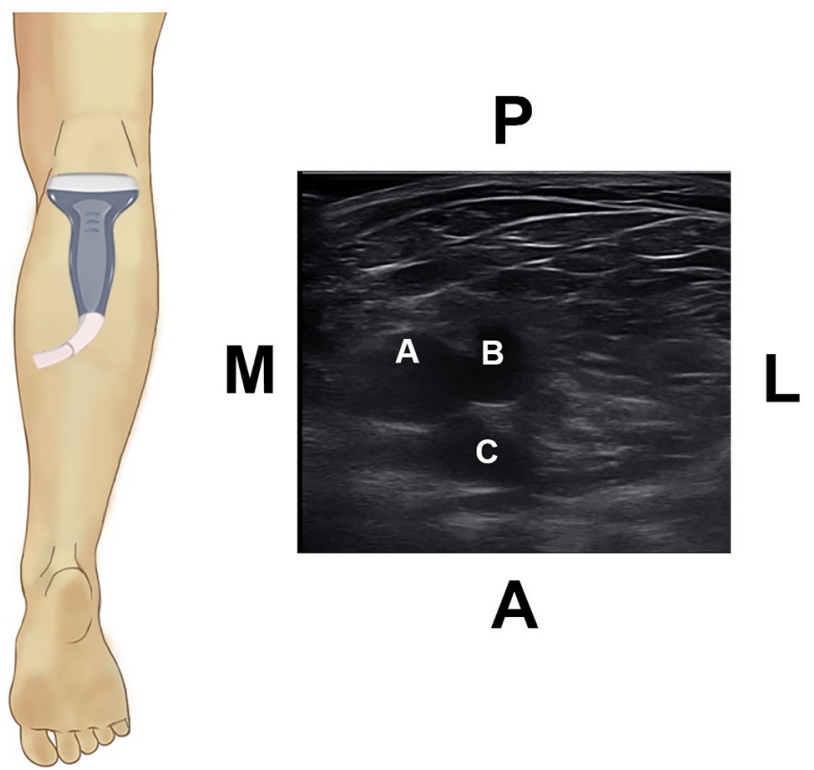

A

Fig. 3 Ultrasonographic image of the deep calf veins, showing the PPTV (A) merging with the ATV (B) to form the PoV. The popliteal artery $(\mathrm{PoA})(\mathbf{C})$ is present deep to the veins. $\mathrm{P}$, posterior; A, anterior; $\mathrm{M}$, medial; L, lateral

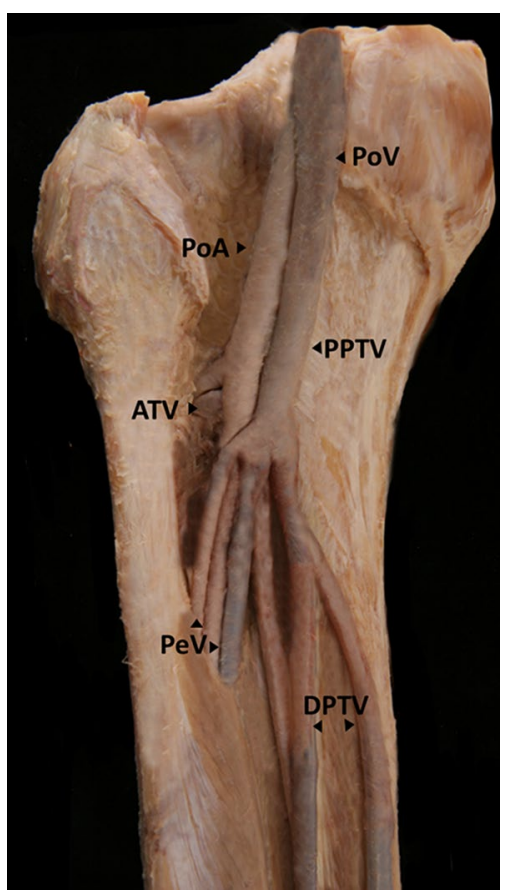

Fig. 4 Image of the left side of a deep calf vein and an artery specimen

\section{Surface area}

The popliteal vein had the largest surface area, at $0.432 \pm 0.092 \mathrm{~cm}^{2}$, followed by the proximal posterior tibial

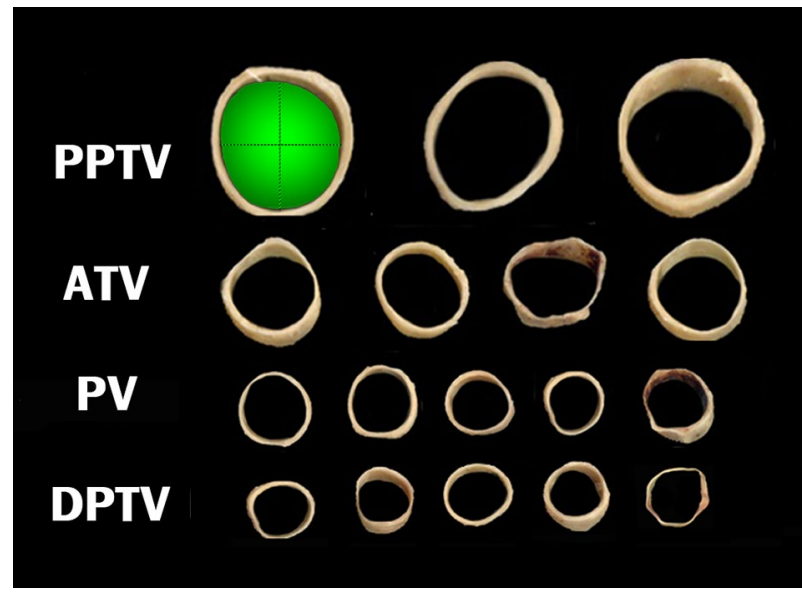

Fig. 5 Sliced venous specimens that were scanned and then measured using Image J. The green shaded area represents the surface area, and dotted lines represent the horizontal and longitudinal diameters of the PPTV

vein $\left(0.312 \pm 0.093 \mathrm{~cm}^{2}\right)$, anterior tibial vein $(0.213 \pm 0.091$ $\left.\mathrm{cm}^{2}\right)$, peroneal vein $\left(0.091 \pm 0.062 \mathrm{~cm}^{2}\right)$, and distal posterior tibial vein $\left(0.081 \pm 0.065 \mathrm{~cm}^{2}\right)$.

\section{Discussion}

The main objective of DVT treatment is to inhibit the expansion of the thrombus load within the affected vein and avoid subsequent pulmonary embolism. PTS also needs to be prevented, which is triggered by valvular insufficiency and an inadequately treated or untreated venous obstruction $[6,21]$.

Previous research has shown that a residual thrombus and recurrent DVT are powerful predictors of subsequent PTS. It was demonstrated that there is a direct correlation between insufficient thrombus lysis and the long-term development of PTS [11]. Another study demonstrated that improving the venous valvular function by resolving thrombus can reduce the probability of PTS [12].

Conventional treatment with systemic anticoagulation was found to result in problems with residual thrombus, and so the treatment standard was changed to combination therapy with CDT [19]. Combined treatment with systemic anticoagulation and CDT resulted in fewer remaining thrombi and reduced the recurrence of thrombosis, while at the same time decreasing the prevalence of PTS [24]. Fleck et al. proposed that combined therapy with CDT is more advantageous in younger patients and those with a more substantial thrombus load [10].

The popliteal vein is currently the most frequently utilized for CDT $[6,23]$. However, the retrograde approach of CDT via the popliteal vein has a reported downside of residual thrombus after CDT, particularly in the inferior 
popliteal vein $[5,7,16]$. Furthermore, the patient needs to be in the prone position during the retrograde approach, and the associated positional difficulties are particularly problematic in the presence of severe obesity, paralysis, or fractures [28].

This situation has led to the antegrade approach being commonly used as an alternative method from the posterior tibial vein. A previous clinical study demonstrated that there were more sequelae during short-term follow-up for enhanced lysis of the distal segments of a thrombosis when the approach was from the posterior tibial vein. Since the approach is in the direction of the blood flow and results in less damage to the valves and vein walls, the posterior tibial vein was suggested as the best venous entry point for CDT [4].

Bendix et al. reported that the long-term outcomes were better for posterior tibial venous access than for popliteal venous access [5]. However, those authors indicated that great care is required when performing antegrade approaches due to the smaller diameter of the veins. Our research has revealed that the small diameter of the distal posterior tibial vein limits its utilization, and so we recommend the proximal posterior tibial vein as the best venous entry point.

Despite antegrade approaches having many advantages over retrograde approaches, they have impediments for clinicians associated with variations in the posterior tibial vein [29]. Yi \& Kim recently classified the posterior tibial vein into the proximal posterior tibial vein and the distal posterior tibial vein based on significant anatomical differences regarding the merging of other veins. The distal posterior tibial vein merges with the peroneal vein and turns into the proximal posterior tibial vein, while the proximal posterior tibial vein merges with the anterior tibial vein and becomes the popliteal vein [17]. Most veins have two accompanying veins in addition to the corresponding artery. However, those authors found that the popliteal vein had one accompanying vein, distal posterior tibial vein had two or three, the proximal posterior tibial veins had one or two accompanying veins, the anterior tibial vein had two accompanying veins, and the peroneal vein had two accompanying veins.

In conclusion, CDT should be carried out by trained physicians with sound knowledge of the venous structures as well as the diameters of the approached veins. This study measured the surface areas of deep calf veins in order to identify the most-appropriate veins for CDT. The obtained results suggest that the distal posterior tibial vein is not appropriate for CDT due its larger anatomical variations and smaller surface area compared with other veins. We also suggest that the peroneal vein should not be used, since it has the second-smallest surface area of all the deep calf veins. We further suggest that the proximal tibial vein and the anterior tibial vein could be considered as ideal veins for CDT.
The present findings indicate that a thorough understanding of the variations and diameters of deep calf veins is necessary for guiding practitioners who are selecting an antegrade approach for CDT.

Acknowledgements The authors sincerely thank those who donated their bodies to science so that anatomical research could be performed. Results from such research can potentially increase mankind's overall knowledge that can then improve patient care. Therefore, these donors and their families deserve our highest gratitude. The authors thank Eun-Byul Yi from Eonbuk elementary school for illustrations. This work was supported by the National Research Foundation of Korea (NRF) grant funded by the Korean government (MSIP) (NRF-2019R1C1C1010776).

Author contribution KHY: research concept, study design, visualization and writing of the manuscript. HJK: reviewing/editing a draft of the manuscript and supervision of the manuscript.

Funding Not applicable.

\section{Declarations}

Conflict of interest The author(s) declare no competing interests.

\section{References}

1. Adachi B (1933) Anatomie der Japaner 1, 1. Verl. d. Kaiserl.Japan. Univ. zu Kyoto, Kyoto

2. Adachi B (1940) Anatomie der Japaner, 222 2. Verl. der Kaiserlich-Japan Univ., Kyoto

3. Alhalbouni S, Hingorani A, Shiferson A, Marks N, Ascher E (2011) Infra-popliteal deep venous thrombi and the risk of symptomatic pulmonary embolism in hospitalized patients. Vascular 19(1):29-33

4. Armon MP, Whitaker SC, Tennant WG (1997) Catheter-directed thrombolysis of iliofemoral deep vein thrombosis. A new approach via the posterior tibial vein. Eur J Vasc Endovasc Surg 13(4):413-416

5. Bendix SD, Nolan R, Banipal S, Oppat WF (2019) Posterior tibial vein approach to catheter-directed thrombolysis for iliofemoral deep venous thrombosis. J Vasc Surg Venous Lymphat Disord 7(5):629-634

6. Comerota AJ, Grewal N, Martinez JT, Chen JT, Disalle R, Andrews L, Sepanski D, Assi Z (2012) Postthrombotic morbidity correlates with residual thrombus following catheter-directed thrombolysis for iliofemoral deep vein thrombosis. J Vasc Surg 55(3):768-773

7. Duan PF, Ni CF (2016) Randomized study of different approaches for catheter-directed thrombolysis for lower-extremity acute deep venous thrombosis. J Formos Med Assoc 115(8):652-657

8. Enden T, Klow NE, Sandvik L, Slagsvold CE, Ghanima W, Hafsahl G, Holme PA, Holmen LO, Njaastad AM, Sandbaek G, Sandset PM, CaVen TSG (2009) Catheter-directed thrombolysis vs. anticoagulant therapy alone in deep vein thrombosis: results of an open randomized, controlled trial reporting on short-term patency. J Thromb Haemost 7(8):1268-1275

9. Enden T, Sandvik L, Klow NE, Hafsahl G, Holme PA, Holmen LO, Ghanima W, Njaastad AM, Sandbaek G, Slagsvold CE, Sandset PM (2007) Catheter-directed Venous Thrombolysis in acute iliofemoral vein thrombosis-the CaVenT study: rationale 
and design of a multicenter, randomized, controlled, clinical trial (NCT00251771). Am Heart J 154(5):808-814

10. Fleck D, Albadawi H, Shamoun F, Knuttinen G, Naidu S, Oklu R (2017) Catheter-directed thrombolysis of deep vein thrombosis: literature review and practice considerations. Cardiovasc Diagn Ther 7(Suppl 3):S228-S237

11. Grewal NK, Martinez JT, Andrews L, Comerota AJ (2010) Quantity of clot lysed after catheter-directed thrombolysis for iliofemoral deep venous thrombosis correlates with postthrombotic morbidity. J Vasc Surg 51(5):1209-1214

12. Haig Y, Enden T, Grotta O, Klow NE, Slagsvold CE, Ghanima W, Sandvik L, Hafsahl G, Holme PA, Holmen LO, Njaaastad AM, Sandbaek G, Sandset PM, CaVen TSG (2016) Post-thrombotic syndrome after catheter-directed thrombolysis for deep vein thrombosis (CaVenT): 5-year follow-up results of an open-label, randomised controlled trial. Lancet Haematol 3(2):e64-71

13. Jaff MR, McMurtry MS, Archer SL, Cushman M, Goldenberg N, Goldhaber SZ, Jenkins JS, Kline JA, Michaels AD, Thistlethwaite P, Vedantham S, White RJ, Zierler BK, American Heart Association Council on Cardiopulmonary CCP, Resuscitation, American Heart Association Council on Peripheral Vascular D, American Heart Association Council on Arteriosclerosis T, Vascular B (2011) Management of massive and submassive pulmonary embolism, iliofemoral deep vein thrombosis, and chronic thromboembolic pulmonary hypertension: a scientific statement from the American Heart Association. Circulation 123(16):1788-1830

14. Kahn SR, Galanaud JP, Vedantham S, Ginsberg JS (2016) Guidance for the prevention and treatment of the post-thrombotic syndrome. J Thromb Thrombolysis 41(1):144-153

15. Libertiny G, Hands L (1999) Lower limb deep venous flow in patients with peripheral vascular disease. J Vasc Surg 29(6):1065-1070

16. Liu G, Liu X, Wang R, Ye K, Yin M, Huang X, Lu M, Li W, Lu X, Jiang M (2018) Catheter-directed thrombolysis of acute entire limb deep vein thrombosis from below the knee access: a retrospective analysis of a single-center experience. Catheter Cardiovasc Interv 91(2):310-317

17. Meissner MH (2005) Lower extremity venous anatomy. Semin Intervent Radiol 22(3):147-156

18. Meissner MH, Gloviczki P, Comerota AJ, Dalsing MC, Eklof BG, Gillespie DL, Lohr JM, McLafferty RB, Murad MH, Padberg F, Pappas P, Raffetto JD, Wakefield TW, Society for Vascular S, American Venous F (2012) Early thrombus removal strategies for acute deep venous thrombosis: clinical practice guidelines of the Society for Vascular Surgery and the American Venous Forum. J Vasc Surg 55(5):1449-1462

19. Meissner MH, Wakefield TW, Ascher E, Caprini JA, Comerota AJ, Eklof B, Gillespie DL, Greenfield LJ, He AR, Henke PK, Hingorani A, Hull RD, Kessler CM, McBane RD, McLafferty
R (2007) Acute venous disease: venous thrombosis and venous trauma. J Vasc Surg 46(6):25S-53S

20. Mewissen MW, Seabrook GR, Meissner MH, Cynamon J, Labropoulos N, Haughton SH (1999) Catheter-directed thrombolysis for lower extremity deep venous thrombosis: report of a national multicenter registry. Radiology 211(1):39-49

21. Morris TA (2011) Natural history of venous thromboembolism. Crit Care Clin 27(4):869-884

22. Parisi R, Visona A, Camporese G, Verlato F, Lessiani G, Antignani PL, Palareti G (2009) Isolated distal deep vein thrombosis: efficacy and safety of a protocol of treatment. Treatment of Isolated Calf Thrombosis (TICT) Study. Int Angiol 28(1):68-72

23. Patterson BO, Hinchliffe R, Loftus IM, Thompson MM, Holt PJ (2010) Indications for catheter-directed thrombolysis in the management of acute proximal deep venous thrombosis. Arterioscler Thromb Vasc Biol 30(4):669-674

24. Raju S, Davis M, Martin A (2014) Assessment of residual thrombus after venous thrombolytic regimens. J Vasc Surg Venous Lymphat Disord 2(2):148-154

25. Sillesen H, Just S, Jorgensen M, Baekgaard N (2005) Catheter directed thrombolysis for treatment of ilio-femoral deep venous thrombosis is durable, preserves venous valve function and may prevent chronic venous insufficiency. Eur J Vasc Endovasc Surg 30(5):556-562

26. Singh K, Yakoub D, Giangola P, DeCicca M, Patel CA, Marzouk F, Giangola G (2012) Early follow-up and treatment recommendations for isolated calf deep venous thrombosis. J Vasc Surg 55(1):136-140

27. Vedantham S, Goldhaber SZ, Julian JA, Kahn SR, Jaff MR, Cohen DJ, Magnuson E, Razavi MK, Comerota AJ, Gornik HL, Murphy TP, Lewis L, Duncan JR, Nieters P, Derfler MC, Filion M, Gu CS, Kee S, Schneider J, Saad N, Blinder M, Moll S, Sacks D, Lin J, Rundback J, Garcia M, Razdan R, VanderWoude E, Marques V, Kearon C, Investigators AT (2017) Pharmacomechanical catheterdirected thrombolysis for deep-vein thrombosis. N Engl J Med 377(23):2240-2252

28. Wang H, Qi X, Luo H, Zhang Q, Chen Y, Sun J (2018) Catheterdirected thrombolysis through anterior tibial vein for treating acute extensive deep venous thrombosis. J Vasc Surg Venous Lymphat Disord 6(6):681-688

29. Yi KH, Kim HJ (2020) Is variation in posterior tibial veins a risk factor for deep-vein thrombosis? Clin Anat. https://doi.org/10. 1002/ca.23691

Publisher's Note Springer Nature remains neutral with regard to jurisdictional claims in published maps and institutional affiliations. 